\title{
BSPD Outstanding Innovation Award
}

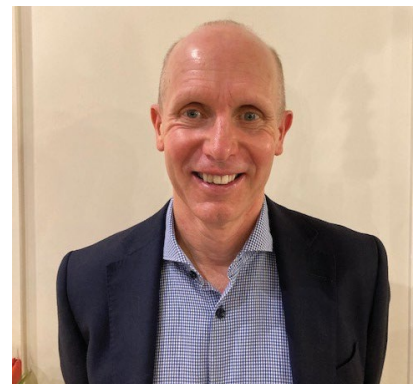

Simon Hearnshaw, on behalf of the North Yorkshire and Humber Local Dental Network (LDN), is the winner of the British Society of Paediatric Dentistry's (BSPD's) Outstanding Innovation Award 2021.

As Chairman of the North Yorkshire and Humber LDN, Dr Hearnshaw (pictured) impressed the judges with the 'In Practice Prevention' (IPP) scheme that ensured thousands more children in the region got access to preventive treatment in a dental practice.

The IPP programme was targeted at deprived areas where oral health need is greatest and at children with the highest levels of disease. A group of dental practices were funded, through a bespoke flexible commissioning approach, to offer more appointments to children.

Health Education England provided training for dental nurses to develop additional skills, such as applying fluoride varnish. The programme treated more than 17,000 children over 2.5 years. Access improved, as did fluoride varnish application rates. There was also a shift of treatment activity towards a more preventive approach.

BSPD's judges said 'With his In Practice Prevention programme working with North Yorkshire and Humber LDN, Simon Hearnshaw addressed BSPD's key aims: improving the oral health of children, reducing oral health inequalities, and upskilling of dental teams to drive up prevention.

'We can see the potential for this initiative to be rolled out nationally and have a longlasting effect. Simon is 2021's worthy winner.'

Dr Hearnshaw said: 'This recognition is shared by all the practices which got on board as well as the brilliant dental nurses who delivered the care, helping to make a difference to thousands of children. Our programme demonstrated that, with the right approach, flexible commissioning works'.

BSPD is delighted by the variety in this year's entries which included Jo Dawson's Raisin Awareness Campaign; the Tooth FaiRead blog from Dayna Rosenthal and Nikita Vasria-Shah; and Katie Davies' HaBox, a dental product subscription box for children.

\section{Rapid review of AGPs guidance reassessed}

The Scottish Dental Clinical Effectiveness Programme (SDCEP) published Mitigation of aerosol generating procedures in dentistry - a rapid review in September 2020. ${ }^{1}$

It was noted at that time that this was a living document and that the Working Group would continue to assess emerging evidence in the following months. The Working Group met again on 13 January 2021. New members present included the Chair of the SAGE Environment and Modelling Group, who is an expert in healthcare ventilation, and representatives from the National Physical Laboratory.

The literature search used for the rapid review has been updated and the results screened for relevance to the review questions. Appraisal is ongoing but, to date, no new evidence has been identified that changes the conclusions of the rapid review.

The Working Group considered the implications of the substantial increase in prevalence of COVID-19 infections in recent months, ${ }^{2}$ the emergence of more transmissible variants of SARS-CoV-2, no reports of transmission associated with dental care, the greater availability of testing and the vaccination programme that has recently commenced. The Working Group agreed that at present, despite these developments, the agreed positions and other conclusions within the rapid review remain unchanged.

The Working Group was keen to re-emphasise the importance of staff and patients continuing to adhere to the precautions specified in current national guidance, including:

- Reviewing all stages of the patient journey to assess adherence to procedures and any improvements required

- Rigorous use of face coverings/masks and application of social distancing measures in clinical and nonclinical areas, including staff rooms

- Use of the correct level of PPE

- Individual patient risk assessment to determine the appropriate COVID-19 care pathway as described in the PHE Infection Prevention and Control (IPC) guidance ${ }^{3}$

o The IPC guidance describes high, medium and low risk care pathways. For a patient to be in the low risk pathway, a negative PCR test is required, and the patient must have followed self-isolation advice. A lateral flow test is not sufficient unless the patient is part of a regular formal NHS testing plan and remains negative and asymptomatic o While vaccination will provide protection for the vaccinated individual, this is not $100 \%$ and it is also unknown whether vaccination prevents onward transmission. Therefore, the same precautions are required for both vaccinated and unvaccinated patients and staff

o Screening questions should be consistent with current triage criteria, including quarantine requirements following international travel ${ }^{4}$

- Adequate ventilation in both clinical and non-clinical areas.

\section{References}

1. SDCEP. Rapid Review of Aerosol Generating Procedures in Dentistry. Updated 25 January 2021. Available at https://www.sdcep.org.uk/published-guidance/covid19-practice-recovery/rapid-review-of-agps/ (accessed February 2021).

2. UK Government. Cases in the United Kingdom. Available at https://coronavirus.data.gov.uk/details/ cases.

3. Public Health England. COVID-19: infection prevention and control (IPC). 10 January 2020, updated 21 January 2021. Available at https://www.gov.uk/government/ publications/wuhan-novel-coronavirus-infectionprevention-and-control (accessed 13 February 2021).

4. NIPCM. Scottish COVID-19 Infection Prevention and Control Addendum for Acute Settings http://www. nipcm.hps.scot.nhs.uk/scottish-covid-19-infectionprevention-and-control-addendum-for-acutesettings/\#a2702 (accessed 13 February 2021). 